\title{
Extending a dynamic friction model with nonlinear viscous and thermal dependency for a motor and harmonic drive gear
}

\author{
Sebastian Wolf and Maged Iskandar
}

\begin{abstract}
In robotic actuation a well identified and modeled friction behavior of the actuator components helps to significantly improve friction compensation, output torque estimation, and dynamic simulations. The friction of two components, i.e. a brush-less DC motor and a harmonic drive gear (HD) is investigated in order to build an accurate dynamic model of the main actuator of the arms of the humanoid David namely the DLR Floating Spring Joint (FSJ). A dedicated testbed is built to precisely identify input and output torques, temperatures, positions, and elasticities of the investigated components at a controlled environment temperature. Extensive test series are performed in the full velocity operating range in a temperature interval from 24 to $50{ }^{\circ} \mathrm{C}$. The nonlinear influences of velocity and temperature are identified to be dominant effects. It is proposed how to include these nonlinear velocity and temperature dependencies into a static and a dynamic friction model, e.g. LuGre. Dynamic models of the motor and HD are built with the proposed method and experimentally evaluated. The new models are compared to friction models with linear dependencies and show a significant improvement of correspondence with reality.
\end{abstract}

\section{INTRODUCTION}

An accurate friction model can improve robotic systems in many ways. It is commonly used for energy efficiency calculation [1], a more reliable dynamic simulation, or to improve the control performance of the overall system. The latter is typically introduced with an additional term in the feed-forward part of the control structure to eliminate the unwanted frictional effects by model based friction compensation [2]. This model is used widely in different research areas such as fault detection and condition monitoring [3], [4]. Furthermore, it can improve the quality of an output torque estimation.

The presented work aims to dynamically model the DLR Floating Spring Joint (FSJ) which is the main arm actuator unit of the German Aerospace Centers (DLR's) Humanoid robot David, see Fig 11. In order to have a complete understanding and an efficient control over the FSJ, an accurate dynamical model is required. The model has to be suitable for a discrete time step real-time model, that is implemented in Matlab/Simulink. In the development a close correspondence with the real system rivals with reasonable computational costs.

All friction contacts in our system are lubricated friction contacts in gears and roller bearings, which are also the most common friction contacts in robotic actuation. As a consequence the focus of this work is on mechanical losses

All authors are with the DLR - German Aerospace Center, Robotics and Mechatronics Center, Institute of Robotics and Mechatronics, Wessling, Germany. Email: sebastian.wolf@dlr.de

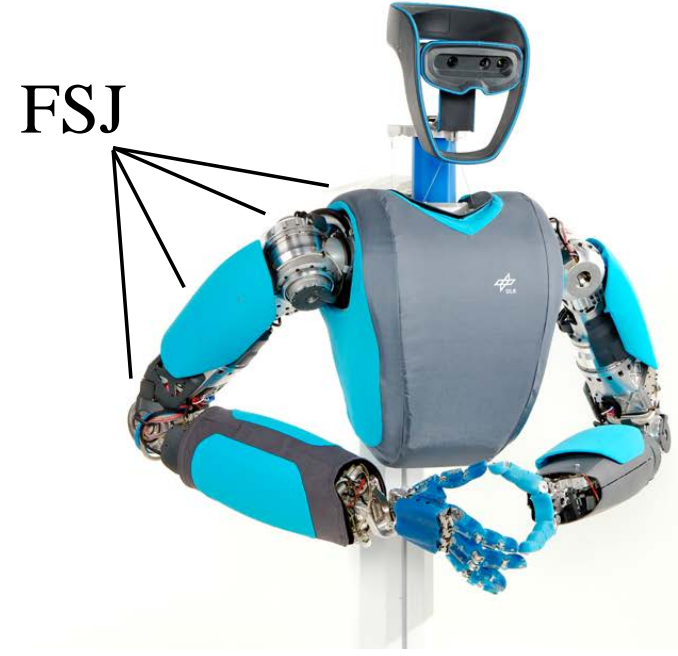

Fig. 1: Dynamic friction models are developed to model the FSJ, which is the main actuator unit of the arms of DLR David.

that are mostly caused by the friction in the main drive train, i.e. the main motor and the main harmonic drive gear (HD). The HD is considered as an essential element in the FSJ and is also extensively employed in most other robotic applications with space and weight constraints.

Nevertheless, the HD exhibits nonlinear behavior and frictional dissipation limiting its performance. As reported in the manufacturers catalogs [5] typical friction losses are greater than twenty percent from the transmitted power. Moreover, the nonlinearity of the viscous behavior of the friction is one of the most important attributes when modeling the losses of the robot joints with a harmonic drive gear. Furthermore, it showed an improvement in the model integrity when the nonlinear frictional losses were added [6].

Introducing a Coulomb friction model for the the zero crossing causes inaccuracies, oscillations and model instability with discrete time step solvers, caused by its jump discontinuity. Continuous and varying time step solvers, which could handle discontinuous behavior better, can not be used for real-time robot control. For sake of simplicity most of the current dynamic robotic actuator models consider modeling the friction with linear viscous part, which neither matches roller bearing friction nor harmonic drive gear friction closely. Roller bearing friction was investigated in [7], [8] and found to have a strongly nonlinear viscous friction dependency. They modeled the viscous part with an exponential function and approximated it with a first and 
third order polynomial expression.

The harmonic drive gear is already extensively studied and various effects identified. Seyfferth et al. as well as Tuttle et al. observed and modeled nonlinear transmission friction, compliance, and kinematic error [9], [10]. Seyfferth and Tuttle modeled friction as a second and third order polynomial velocity dependency, respectively. Both used a static friction model which will cause serious stability problems when integrated into a fixed step real-time model. Taghirad et al. proposed a transmission model of harmonic drive gear with compliance, position hysteresis, and a Dahl model as a dynamic friction model [11]. However, the friction was identified for only two velocities and the rather old Dahl model has some serious drawbacks, including drift at low velocities, compared to more recent dynamic friction models, e.g. Lund Grenoble model (LuGre) [12], [13]. None of the aforementioned studies on the HD investigated temperature effects. Only Bittencourt et al. [14] investigated temperature dependency in a lumped robot joint of an ABB robot.

In this work we take two typical components of a robotic actuator, i.e. a motor and a HD, and show the effect of nonlinear viscous friction and the nonlinear temperature dependency. An extension to a static and a state of the art dynamic friction model is proposed. Typical humanoid robotic movements with resting, slow, and fast movements should be well modeled. As a result we aim at a good coverage of the zero velocity crossing behavior, at slow velocities, and near maximum velocity. Furthermore, the effect of varying temperature is investigated and will be integrated in the same friction model extension. Finally, we want to show the improved behavior compared to commonly used implementations.

The paper is organized as follows. Section $\mathrm{II}$ gives an overview on the FSJ and the underlying standard methods for this work. Section III contains the proposed method. The testbed setup is described in Section [V] and the results are presented in Section $\mathrm{V}$. The conclusions can be found in Section VI.

\section{BACKGROUND}

\section{A. Dynamic Model of the DLR FSJ}

Building an accurate dynamic model of a robotic actuator includes an investigation of the friction. Fig. 2 shows simplified schematic representation for the inertia of the system with the associated velocities and the different friction contacts, which are illustrated in Table \$ (see also [1]).

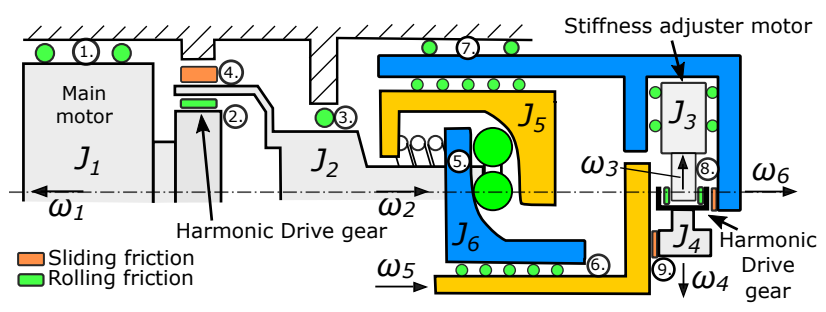

Fig. 2: The FSJ drive train with numbered friction contacts. Inertia $J_{n}$ are lumped and depicted with their velocities $\omega_{n}$.
TABLE I: Contact areas with friction in the FSJ

\begin{tabular}{rrl}
\hline Number & Symbol & Description of friction contact area \\
\hline 1 & $\tau_{\mathrm{r} 1}$ & roller bearing of rotor \\
2 & $\tau_{\mathrm{r} 2}$ & roller bearing of wave generator \\
3 & $\tau_{\mathrm{r} 3}$ & roller bearing of flex spline \\
4 & $\tau_{\mathrm{r} 4}$ & tooth meshing of HD gear \\
5 & $\tau_{\mathrm{r} 5}$ & bearing and roller friction of cam roller \\
6 & $\tau_{\mathrm{r} 6}$ & linear guide of spring mechanism \\
7 & $\tau_{\mathrm{r} 7}$ & roller bearing of output \\
8 & $\tau_{\mathrm{r} 8}$ & friction contacts of stiffness adjuster unit \\
9 & $\tau_{\mathrm{r} 9}$ & tooth meshing of hypoid gear \\
\hline
\end{tabular}

In order to model each friction contact, a detailed component-wise experimental identification would be necessary. This would lead to many complex test-beds with multiple test runs. The identification process can be simplified by grouping the friction contacts with the same movements, load, and temperature. In this case the friction of the different friction contacts can be combined as follows:

$$
\begin{aligned}
\tau_{\mathrm{r}, \text { Main }} & =\tau_{\mathrm{r} 1}+\tau_{\mathrm{r} 2}+\tau_{\mathrm{r} 3}+\tau_{\mathrm{r} 4} \\
\tau_{\mathrm{r}, \text { Spring }} & =\tau_{\mathrm{r} 5}+\tau_{\mathrm{r} 6} \\
\tau_{\mathrm{r}, \text { Out }} & =\tau_{\mathrm{r} 7} \\
\tau_{\mathrm{r}, \text { Adj }} & =\tau_{\mathrm{r} 8}+\tau_{\mathrm{r} 9} .
\end{aligned}
$$

With combined friction torques, the spring torque $\tau_{\text {Spring, }}$, and the HD gear ratio $i_{1}$, the dynamic motion equations can be expressed for the main drive train as

$$
\begin{gathered}
\underbrace{\left(J_{1} i_{1}^{2}+J_{2}\right)}_{J_{\text {main }}} \ddot{\theta}_{\mathrm{j}}+\tau_{\mathrm{r}, \text { Main }}+\tau_{\mathrm{r}, \text { Spring }}+\tau_{\text {Spring }}=\tau_{\mathrm{m}} \\
J_{6} \ddot{q}_{\mathrm{j}}+\tau_{\mathrm{r}, \text { Out }}-\tau_{\mathrm{r}, \text { Spring }}-\tau_{\text {Spring }}=\tau_{\mathrm{j}} .
\end{gathered}
$$

The motion equation of the stiffness adjuster unit is

$$
\underbrace{\left(J_{3}\left(i_{2} i_{3}\right)^{2}+J_{4} i_{3}^{2}+J_{5}\right)}_{J_{\mathrm{adj}}} \ddot{q}_{\mathrm{adj}}+\tau_{\mathrm{r}, \mathrm{Adj}}=-\tau_{\text {Spring }}-\tau_{\mathrm{a}} i_{1} i_{2},
$$

with the stiffness adjuster motor torque $\tau_{\mathrm{a}}$ and the transmission ratios $i_{2}$ and $i_{3}$ of the two staged gear.

\section{B. Cross-correlation Method}

The cross-correlation method is used as a tool for eliminating the non-relevant parts of the signal to identify the motor inertia $J_{1}$ separately. Furthermore, for extracting the part that corresponds to the inertial torque without friction effects, the correlation between the acceleration $\ddot{\theta}(t)$ and the motor current $i_{\mathrm{m}}$ is provided for the interval $t \in[0, \eta]$ as

$$
\int_{0}^{\eta} k_{\mathrm{t}} i_{\mathrm{m}}(t) \ddot{\theta}(t) d t=\int_{0}^{\eta} \tau_{\mathrm{r}}(\dot{\theta}(t)) \ddot{\theta}(t) d t+J_{1} \int_{0}^{\eta} \ddot{\theta}^{2}(t) d t
$$

The above equation describes the principle of inertia identification method that has been used in [15]. The left hand side term of the equation represents the correlation between the motor current and the acceleration. With the Newton-Euler formalism we get the right hand side, which includes the correlation between given friction torque and the acceleration in $t \in[0, \eta]$. We can eliminate the influence of the friction torque by obtaining the angular velocity equal 
to zero at the beginning and the end of the experiment $\dot{\theta}(0)=\dot{\theta}(\eta)=0$, such that

$$
\int_{0}^{\eta} \tau_{\mathrm{r}}(\dot{\theta}(t)) \frac{\dot{\theta}}{d t} d t=\int_{\dot{\theta}(0)}^{\dot{\theta}(\eta)} \tau_{\mathrm{r}}(\dot{\theta}) d \dot{\theta}=\int_{(0)}^{(0)} \tau_{\mathrm{r}}(\dot{\theta}) d \dot{\theta}=0 .
$$

In Equations (4) and (5) the common component of the current and acceleration is emphasized, while the uncommon component is suppressed. With the friction torque tending to zero, Equation (4) can be rewritten as:

$$
J_{1}=\frac{\int_{0}^{\eta} k_{\mathrm{t}} i_{\mathrm{m}}(t) \ddot{\theta}(t) d t}{\int_{0}^{\eta} \ddot{\theta}^{2}(t) d t} .
$$

\section{Static Friction Model}

Many empirical and physically-motivated friction models have been proposed with different grades of detail in order to describe the nonlinearity of the interaction between two surfaces. Usually they are referred to as a classical, or static friction model. They represent the basic friction phenomena in the sliding regime. The typical description of the friction torque is a combination of certain aspects of the friction force, such as static friction, Coulomb friction, viscous friction and Stribeck effect [16]. For the rotating parts of the FSJ, the static friction models can be composed differently among the previously mentioned friction phenomena. A common form of the static friction model is expressed as

$$
\begin{aligned}
\tau_{\mathrm{r}, \mathrm{s}}(\dot{\theta}) & =g(\dot{\theta})+s(\dot{\theta}) \\
g(\dot{\theta}) & =\operatorname{sign}(\dot{\theta})\left(F_{\mathrm{c}}+\left(F_{\mathrm{s}}-F_{\mathrm{c}}\right) e^{-\left|\dot{\theta} / v_{\mathrm{s}}\right|^{\delta_{\mathrm{s}}}}\right),
\end{aligned}
$$

but generally static friction can be modeled as an arbitrary function. The function $s(\dot{\theta})$ expresses the velocity strengthening that is well known as viscous friction, and typically it is linear proportional to the relative velocity as $s(\dot{\theta})=f_{\mathrm{v}} \dot{\theta}$ with linear viscous coefficient $f_{\mathrm{v}}$. The function $g(\dot{\theta})$ contributes as the velocity weakening of the friction torque. The function $g(\dot{\theta})$ is alternatively called the Stribeck curve because it captures the Stribeck effect, where $F_{\mathrm{c}}$ is Coulomb friction, $F_{\mathrm{s}}$ is static or stiction friction, $v_{\mathrm{s}}$ is Stribeck velocity, and $\delta_{\mathrm{s}}$ is the exponent parameter of the Stribeck nonlinearity. In the Gaussian parametrization as used in [16] and [17] the exponent parameter is $\delta_{\mathrm{s}}=2$. Parameters of the total friction torque $\tau_{\mathrm{r}, \mathrm{s}}$ can be identified easily using the static map between the friction torque and the relative velocity.

\section{Dynamic Friction Model (LuGre)}

Particularly while crossing zero velocity a smooth and better description of the friction than in the static friction model is important. Following this demand, the static friction model can be extended to a dynamic friction model. The LuGre model was selected among the generalized empirical friction models as it is widely used in the robotics community and promises accurate results. The LuGre model exhibits a good representation of the friction phenomena referring to the experimental observations. It can capture phenomena such as pre-sliding, varying break-away force, stick-slip, and the friction lag. The model uses the bristles concept to reproduce the spring like behavior within a small displacement. It can be described as the dynamic friction torque

$$
\begin{aligned}
\tau_{\mathrm{r}, \mathrm{d}} & =\sigma_{0} z+\sigma_{1} \dot{z}+s(\dot{\theta}), \\
\dot{z} & =\dot{\theta}-\sigma_{0} \frac{|\dot{\theta}|}{g(\dot{\theta})} z,
\end{aligned}
$$

where $z$ is the internal friction state and can be interpreted as the average deflection of the micro bristles, $\sigma_{0}$ is the bristle stiffness, and $\sigma_{1}$ is the micro-damping coefficient. The steady state friction torque is given by Equation (7). The structure of LuGre model gives the ability to model arbitrary steady state friction behavior in the sliding regime. In fact we use this property to use a nonlinear function to describe the gross sliding regime [18]. The identification of the parameters of LuGre model are carried out in two different regimes, the pre-sliding and the gross sliding phases.

\section{PROposed Methods}

\section{A. Friction Model Refinement}

The proposed refinement for the friction models addresses the gross sliding regime. Both effects, the nonlinear viscous friction as well as the nonlinear temperature dependency mainly affect the gross sliding regime. As a result, additional parameters need to be experimentally identified only for the gross sliding regime. Furthermore, it makes it easy to integrate them into state of the art dynamic friction models, such as the LuGre or Generalized-Maxwell-Slip [19]. However, we want to focus in this publication on the LuGre model. As mentioned before, we modify $s(\dot{\theta})$ of Equation (7) with an according nonlinear term.

\section{B. Nonlinear Viscous Friction}

The choice of an expression that accurately describes the viscous part of a static friction model could be arbitrarily formulated depending on the system behavior, e.g. [16].

We investigate the following cases for the velocity strengthening function to improve the representation of the gross sliding (elasto-hydrodynamic lubrication) regime:

Case 1 : Linear velocity strengthening:

$$
s(\dot{\theta})=f_{\mathrm{v}} \dot{\theta},
$$

with the relative velocity coefficient $f_{\mathrm{v}}$.

Case 2: An exponential shaping factor $\delta_{\mathrm{v}}$ in the nonlinearity of viscous part of the static model:

$$
s(\dot{\theta})=f_{\mathrm{v}} \operatorname{sign}(\dot{\theta})|\dot{\theta}|^{\delta_{\mathrm{v}}} .
$$

Case 3: A third order polynomial in order to cover a wider operating range and map nonlinearity:

$$
s(\dot{\theta})=f_{\mathrm{v} 1} \dot{\theta}+f_{\mathrm{v} 2} \operatorname{sign}(\dot{\theta}) \dot{\theta}^{2}+f_{\mathrm{v} 3} \dot{\theta}^{3} .
$$

The three cases are depicted in Fig. 3 .

Lubricated friction is mainly dependent on the amount of lubricant, its type, and its viscosity, which is in most cases strongly dependent on the temperature. 


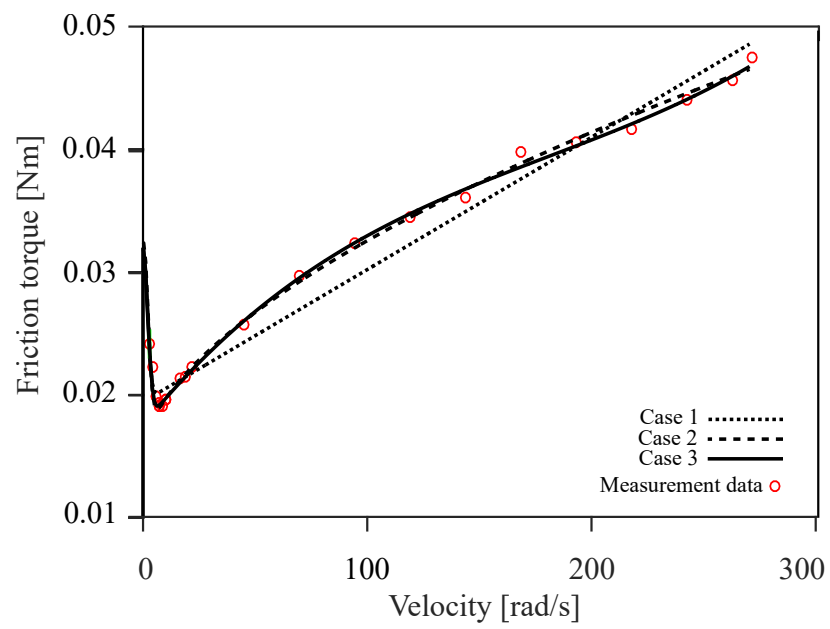

Fig. 3: The representation for the velocity strengthening function $s(\dot{\theta})$. In case 1 the viscous part of the friction has linear relationship with the relative velocity while in case 2 and 3 its approximated by nonlinear functions.

\section{Nonlinear Temperature Dependency}

Temperature affects the behavior of the friction due to the change in the contact point properties as well as the lubricant viscosity. The data sheets of the manufacturer of the harmonic drive gear show that the efficiency is strongly temperature dependent [5]. This correlates with the experimental data of this work, as shown in Sec. V-C

As a consequence, the friction modeling of the FSJ according to Section III-B is only valid at fixed temperature values. In principal the steady state characteristics of the friction is fully described by Equation (7) assuming $s(\dot{\theta})$ can be any function, e.g. one of cases 1 to 3 . This expression can be used to represent the friction at different fixed values of temperature. For this reason an additional formulation with extra parameters is needed in order to map the temperature effect. However, there is no standard way of integration but the temperature effect has to be observed experimentally and possibly expressed by polynomial approximation. In case of the HD it is more difficult to model the temperature nonlinearity as it has different levels of nonlinearity over the velocity range. Therefore, a configurable general approximation method like neural network could be used as an alternative to model different levels of static nonlinearity. The total friction torque as a function of velocity and temperature $T$ can be written as a general function

$$
\tau_{\mathrm{r}, \mathrm{s}, \mathrm{T}}(\dot{\theta}, T)=f(\dot{\theta}, T)+g(\dot{\theta}) .
$$

This function can be expressed by the neural network to change the nominal behavior of the viscous part of the static friction model $\tau_{\mathrm{r}, \mathrm{s}}(\dot{\theta})$ within the temperature range, as it will be seen in Section $\mathrm{V}-\mathrm{C}$

\section{Test Setup}

The mechanical design of the test-bed setup was the most important aspect to precisely identify the main axes joint behavior. Special care has been taken to ensure that

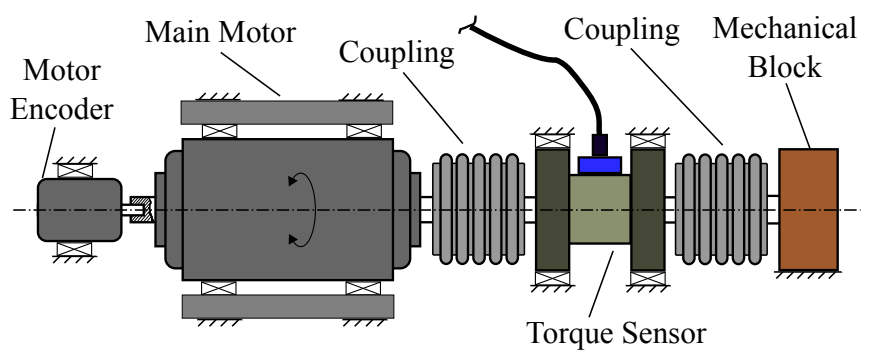

Fig. 5: Special configuration of the experimental setup to identify the motor constant by using a mechanical blocking to have constrained movement.

the system would be valid to measure torques, positions, elasticities, and temperature in different configurations, in order to measure the internal states of the joint actuators. A photo of the drive train experimental setup and its schematic are illustrated in Fig. 4 The main actuator of the experiment setup is a RoboDrive ILM85 servo motor, which is a permanent magnet synchronous motor (PMSM) developed at the Robotics and Mechatronics Center of DLR with maximum rated torque of $1.8 \mathrm{Nm}$. An Elmo Whistle digital servo drive that supports up to $20 \mathrm{~A}$ of continuous current and $1.6 \mathrm{~kW}$ of continuous power, is used to drive the motor. It has been configured and tuned to work in current mode with EtherCAT communication that is connected directly to the real time machine. Ideally the servo drive can be considered as a current source when configured in current or torque mode and the motor supply voltage is $48 \mathrm{VDC}$. The motor is connected rigidly with an incremental encoder from Heidenhain with resolution of 2048 pulses per revolution. The harmonic drive gear in this setup is a CSD 25 of Harmonic Drive AG with gear ratio 1:80 and rated torque of $75 \mathrm{Nm}$, which is also the main gear of the FSJ. The efficiency of this type of harmonic drive gear is a function of ambient temperature, speed, and lubrication conditions [5]. A metal bellows coupling is used for the connection between the motor output shaft and a Lorenz Messtechnik GmbH DR2643 torque sensor with range of $\pm 5 \mathrm{Nm}$. In the low velocity side another DR-2643 torque sensor is connected with range of $\pm 200 \mathrm{Nm}$, which is attached rigidly to an absolute high resolution encoder 25 bit, followed by metal bellow safety coupling to avoid any overload at the output of the harmonic drive gear, and a load inertia, which is used to simulate the robot arm in free-motion. The circular spline of the harmonic drive is fixed to the ground and the output is carried by the flexspline, while the wave generator is the input. This setup corresponds to the FSJ configuration, but please note that for sake of simplicity an ILM85 motor is used instead of the smaller ILM50 of the FSJ. The ILM85 is a bigger version of the main motor of the FSJ, i.e. ILM50, and is the motor of the elastic robot C-Runner [20]. For the final model the same investigations will be performed on the ILM50 motors of David. Considering the motor is free running then the fundamental equation of motion for the DC motor can be 

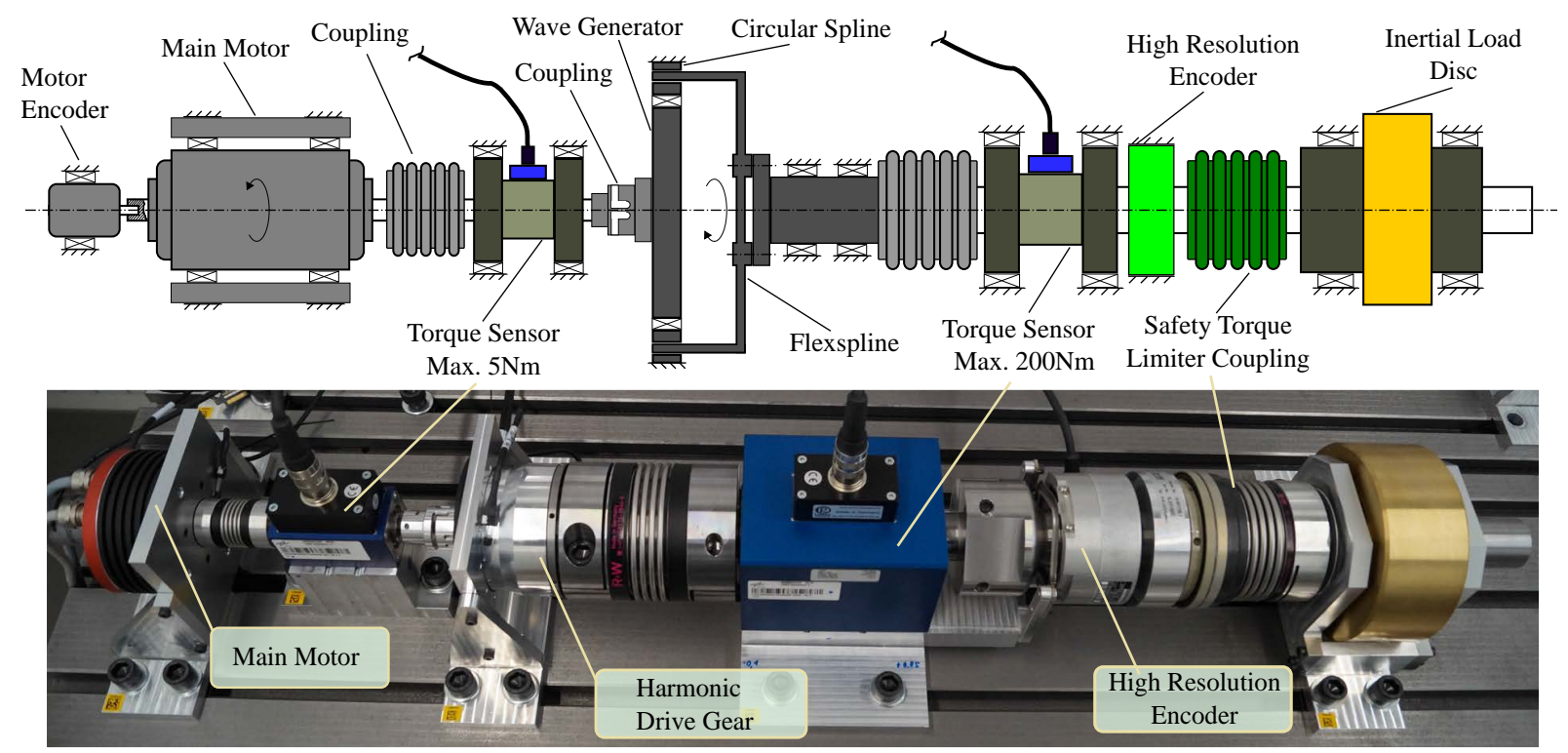

Fig. 4: FSJ principal axes drive train test-bed setup (bottom) and the corresponding schematic diagram (top). The mechanical construction of the test-bed allows to build multi-configuration setups, such as the example depicted in Fig. 5

written as

$$
J_{1} \ddot{\theta}+\tau_{\mathrm{rm}}=u,
$$

where $u$ is the input torque that can be calculated as

$$
u=k_{\mathrm{t}} i_{\mathrm{m}} .
$$

Assuming the motor current is linearly dependent on the output torque, a special modification as shown in Fig. 5 to identify the motor constant $k_{\mathrm{t}}$ has been made. A mechanical block and the intermediate torque sensor with nominal torque of $\pm 5 \mathrm{Nm}$ are used to measure the actual motor torque.

In order to be able to investigate the temperature dependencies on the friction behavior, the temperature has to be controlled. A test-bed cover is used to isolate the surrounding ambient temperature. The ambient temperature of the drive train within the test-bed cover was regulated by a simple standalone on-off controller. Furthermore, additional sensors of type Adafruit MCP9808 are placed on the housing surface of the harmonic drive gear and the motor in order to acquire the temperature.

\section{RESULTS}

\section{A. Motor Constant and Inertia Identification}

An excitation signal with increasing and decreasing sinusoidal was used to identify the torque constant of the main motor of the test-bed in configuration of Fig. 5. The signal was designed to be symmetric and contain different amplitudes. Depicted in Fig. 6 is the actual torque signal that was measured by torque sensor and the motor torque measured by the motor current. Assuming that the measurement data has a Gaussian distribution with zero mean value, the standard least square method is applied to identify the torque constant $k_{\mathrm{t}}=0.1223 \mathrm{Nm} / \mathrm{A}$. The torque constant claimed by manufacturer is $0.13 \mathrm{Nm} / \mathrm{A}$.
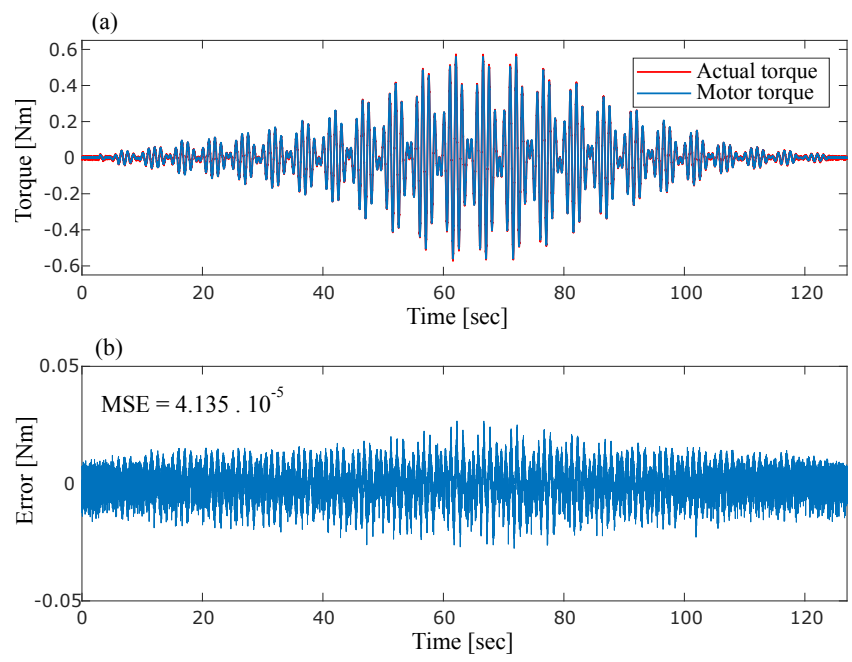

Fig. 6: Actual torque and the motor torque using increasing and decreasing sinusoidal reference signal (a) and the corresponding error plot (b).

The motor inertia is identified with the cross-correlation method, see Sec. III-B Experimental data is collected with the maximum possible acceleration of $\simeq 29000 \mathrm{rad} / \mathrm{s}^{2}$, which excites the maximum possible inertial torque. This leads to a better estimation for the inertia parameter. The velocity is set equal to zero at the start and the end. Using the measurement of the motor current and acceleration, the calculation results in $J_{1}=7.7091910^{-5} \mathrm{kgm}^{2}$. This is slightly less than the motor inertia calculated by CAD, which is $8.010^{-5} \mathrm{kgm}^{2}$.

\section{B. Nonlinear Viscous Friction}

For sake of brevity, the evaluation of nonlinear viscous friction will be shown on the example of the ILM85 motor in this section and the nonlinear temperature dependency on the example of the harmonic drive gear in Sec. $\mathrm{V}-\mathrm{C}$. 
In order to identify the velocity dependency with dedicated variables, it is important to separate the effect in the measurement. A warm up phase is executed to achieve more repeatable conditions and the friction is identified at $30^{\circ} \mathrm{C}$. The experimental data is collected at different constant velocities. The average of the resultant torque is calculated and depicted as circles in Fig. 3. At constant velocities the acceleration is equal to zero, therefore the measured torque is equal to the friction torque.

The three cases of III-B are applied to the ILM85 motor model. The identification of the parameters can be formulated as least squares

$$
\hat{p}=\min _{p} \sum_{i=1}^{N}\left(\hat{\tau}_{\mathrm{r}, \mathrm{s}}(\dot{\theta}, p)-\tau_{\mathrm{r}, \mathrm{s}}(\dot{\theta})\right)^{2},
$$

where the parameter vector $p \in \mathbb{R}^{n_{\mathrm{r}}}$ depends on the considered case. The parameters were initialized heuristically based on the experimental observations and then transferred to a trust-region reflective algorithm available in optimization toolbox of Matlab. The parameters were identified in both directions of motion individually, the average value of each parameter has been taken as the central estimate and the direction dependent values assumed to be the parameters uncertainties as illustrated in Table [II The result of the predicted friction toque is shown in Fig. 3 for the three cases. The previously identified parameters including the torque constant and the motor inertia are integrated into the dynamical friction model and are used to build a dynamical model for ILM85 motor. A chirp signal with increasing frequency and constant amplitude is used as a commanded torque, the same signal is used as an input signal for the model. The comparison between the commanded and the applied torque is presented in Fig. 7(a), where the applied torque is calculated as the product of measured current and motor constant according to Equation (14). Fig. 7(b) shows the open loop velocity response of the models and the measured data. Corresponding to the static friction model presented in Fig. 3 the dependency of friction torque vs. velocity in the dynamic friction of the four investigated models is depicted in Fig. 7( (c). It can be clearly seen that the biggest deviations of the viscous friction model are at low velocities, especially in the zero crossing, where stiction is not part of this approach. This effect can also be seen in the velocity behavior depicted in Fig. 8, where the viscous friction model has big deviations from the real system in the beginning and in each zero crossing. Clearly visible is the good prediction of the stiction phases in the LuGre models. The stiction phases behavior can be further improved with individual stiction level parameters $F_{\mathrm{S}}$ for both directions. However, in order to reduce computational effort and model complexity, a symmetrical approach is used in the presented models.

All LuGre models perform much better, but the mean square error (MSE) of case 3 is the lowest, see Table II] Case 3 has a MSE of $67 \%$ relative to the standard case 1 and only $4 \%$ of the linear viscous friction model. Accordingly
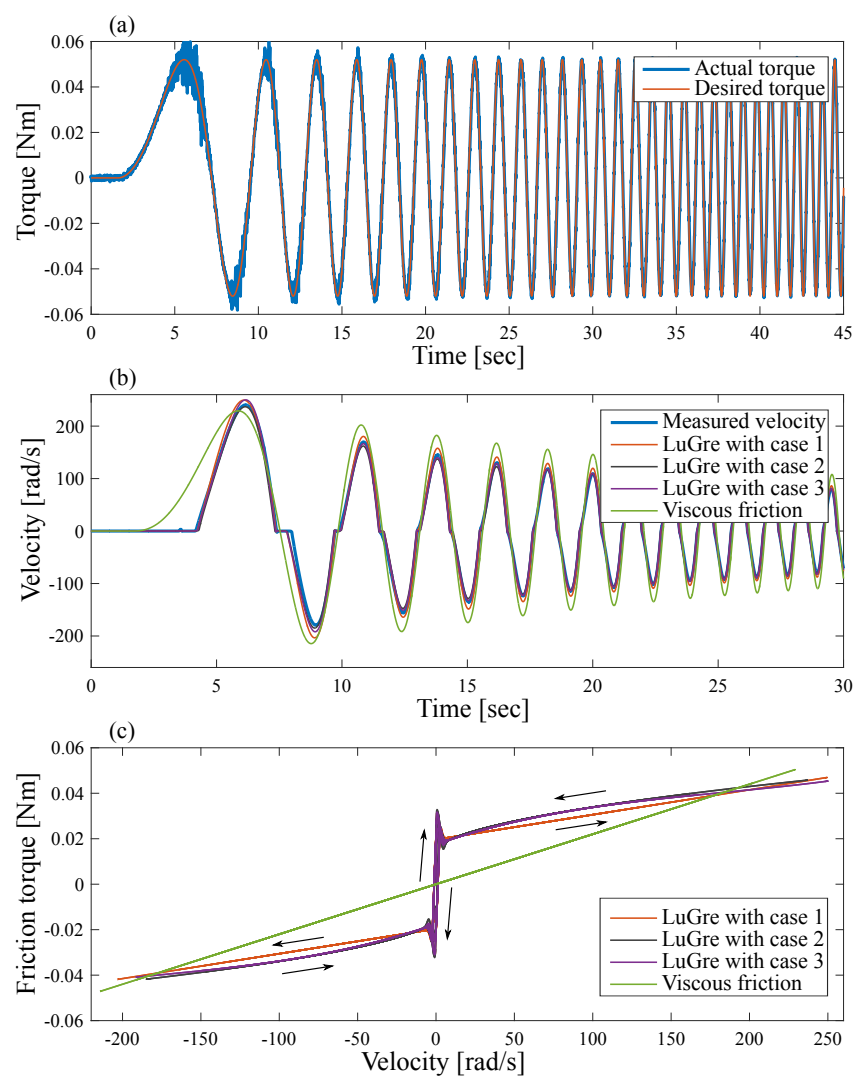

Fig. 7: The evaluation experiment of ILM85 motor using chirp excitation signal. The commanded and actual torques are shown in (a). The measured and modeled velocities as a result of dynamic friction model (LuGre) with standard linear and modified nonlinear viscous behavior in comparison with a pure linear viscous friction are illustrated in (b). The corresponding estimated friction torques are shown in (c).

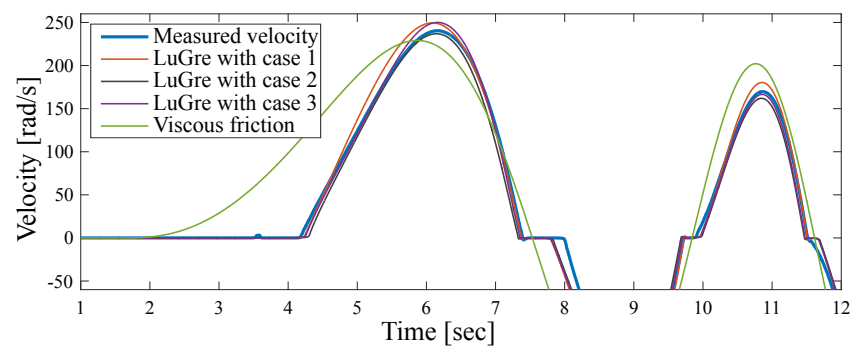

Fig. 8: Clipping of the initial period of the velocity function of Fig. 7b).

case 3 is selected as a modification to express a velocity dependent function $s(\dot{\theta})$ instead of a linear coefficient.

As mentioned before the nonlinear viscous friction identification for the HD is not shown here, but the MSE evaluation found case 2 to suit best.

\section{Nonlinear Temperature Dependency}

The temperature dependency in the FSJ has a big influence on the friction model, as it relies on the harmonic drive gear in the principle axis of movement. Please note that in the following all friction torques are given with respect to the motor/input side of the harmonic drive gear. It can be seen in 
TABLE II: Identified parameters of static curve in case of different $s(\dot{\theta})$ functions for the ILM85 motor

\begin{tabular}{|c|c|c|c|c|c|c|c|}
\hline $\begin{array}{l}\text { Viscous } \\
\text { friction }\end{array}$ & $\begin{array}{c}f_{\mathrm{v}} \\
0.00022\end{array}$ & & & & & & $\begin{array}{l}\text { MSE } \\
1434 \\
\end{array}$ \\
\hline \multirow[t]{2}{*}{ Case 1} & $F_{\mathrm{S}}\left[10^{-2}\right]$ & $F_{\mathrm{C}}\left[10^{-2}\right]$ & $v_{\mathrm{s}}$ & $f_{\mathrm{v}}$ & & & MSE \\
\hline & $3.25 \pm 0.05$ & $1.96 \pm 0.02$ & $2.2 \pm 0.4$ & 0.0001 & & & 81 \\
\hline \multirow[t]{2}{*}{ Case 2} & $F_{\mathrm{s}}\left[10^{-2}\right]$ & $F_{\mathrm{C}}\left[10^{-2}\right]$ & $v_{\mathrm{s}}$ & $f_{\mathrm{V}}\left[10^{-3}\right]$ & $\delta_{\mathrm{v}}\left[10^{-1}\right]$ & & MSE \\
\hline & $3.25 \pm 0.05$ & $1.45 \pm 0.09$ & $2.893 \pm 0.2$ & $1.5 \pm 0.4$ & $5.52 \pm 0.41$ & & 73 \\
\hline \multirow[t]{2}{*}{ Case 3} & $F_{\mathrm{s}}\left[10^{-2}\right]$ & $F_{\mathrm{C}}\left[10^{-2}\right]$ & $v_{\mathrm{s}}$ & $f_{\mathrm{v} 1}\left[10^{-4}\right]$ & $f_{\mathrm{v} 2}\left[10^{-7}\right]$ & $f_{\mathrm{v} 3}\left[10^{-9}\right]$ & MSE \\
\hline & $3.25 \pm 0.05$ & $1.76 \pm 0.005$ & $2.73 \pm 0.28$ & $2.5 \pm 0.5$ & $-9.252 \pm 0.75$ & $1.72 \pm 0.15$ & 54 \\
\hline
\end{tabular}

Fig. 9 that the presence of the harmonic drive gear is highly influenced by the nonlinear static friction characteristics. Therefore, the commonly used static friction models are insufficient to accurately describe the friction behavior due to the nonlinear viscous behavior over the velocity range as well as the dominant temperature dependency. Dedicated intensive experimental study has been carried out to properly collect the data and separate the nonlinearities of both the temperature and velocity.

Due to the nonlinearity of the friction and the rapid change with temperature in case of the harmonic drive, thirty individual experiments have been done for each static friction curve around fixed temperature values. The data was collected by imposing different desired constant velocities and observing the average of the resultant torques and velocities. Fig. 9 shows the experimental data points with the fitted static friction models expressed in Equation (7) with case 2 in the positive directions of motion. The density of measurements was chosen to be higher at low velocities, because the expected nonlinearity of the viscous friction was higher in this region. The accepted uncertainty for the temperature measurements was $\pm 0.1^{\circ} \mathrm{C}$.

A neural network is an alternative to polynomial approaches. It is used to approximate the static nonlinearities of the viscous function $s(\dot{\theta})$ with respect to the thermal dependency. The total friction torque of 12 is then expressed as

$$
\begin{aligned}
\tau_{\mathrm{r}, \mathrm{s}, \mathrm{T}}(\dot{\theta}, T) & =f(\dot{\theta}, T)+g(\dot{\theta}), \\
f(\dot{\theta}, T) & =\xi\left(b+\sum_{1}^{j=3} M\left(b_{j}+\sum_{i=1}^{i=2} x_{i} w_{i j}\right) w_{j}\right), \\
x_{1} & =s(\dot{\theta})=\operatorname{sign}(\dot{\theta})\left(f_{\mathrm{v}}|\dot{\theta}|^{\delta_{\mathrm{v}}}\right), \\
x_{2} & =T,
\end{aligned}
$$

where $\xi$ and $T$ are the neural network function and the temperature, respectively. Further, $w$ and $b$ are the network weight and bias. The neural network function has two inputs and one hidden layer $M$ with 3 neurons. A linear function is used for the output layer while a sigmoid function is used in the neurons of the hidden layer.

We use a combined model to capture the nonlinearities in both velocity and temperature. The model is identified in two steps. At first the two parameters of $x_{1}\left(f_{\mathrm{v}}\right.$ and $\left.\delta_{\mathrm{v}}\right)$ are identified at seven different temperature values, see Fig. 9 . Each static curve is made out of thirty individual experiments over the velocity range that are indicated by markers. It can be clearly seen that the temperature has a significant influence in the static friction behavior. The friction torque at maximum velocity of $275 \mathrm{rad} / \mathrm{s}$ decreased to $42 \%$ within a temperature range from 24 to $50{ }^{\circ} \mathrm{C}$. The identified static models are used to generate about 3000 points per curve to be used as a training set for the neural network. In the second step the universal approximation property of the neural network is used to map the nonlinearity of the temperature dependency. For the neural network the estimated friction torque of the overall model is a function of velocity and temperature.

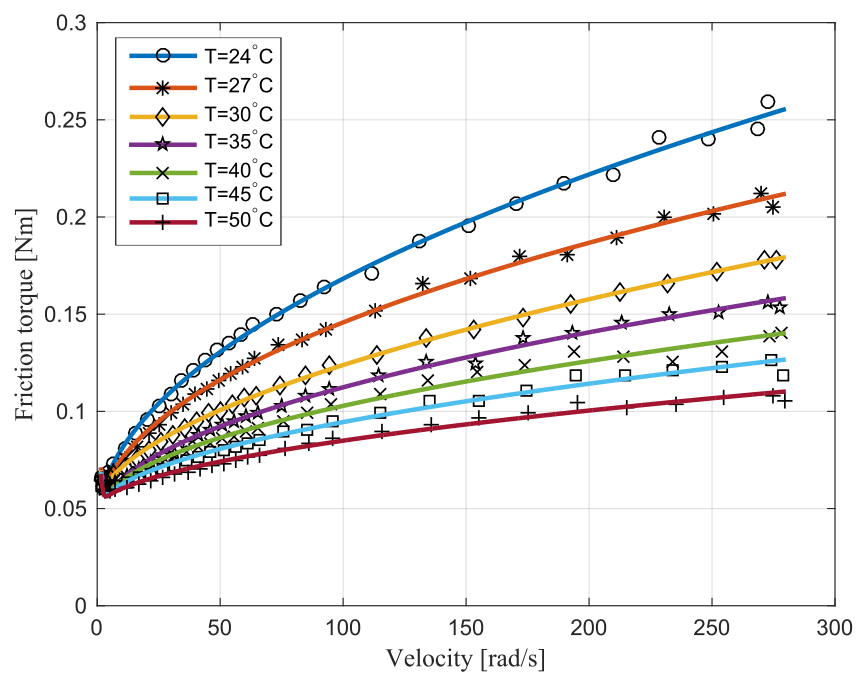

Fig. 9: The static friction curves at different values of operating temperature and velocity. It can be seen easily that the motor side friction torque has nonlinear decreasing behavior with increasing temperature.

\section{Evaluation of the Combined HD Friction Model}

The experimental evaluation of the combined viscous and temperature dependent model has been made to validate the friction behavior with the continuous temperature change. An experiment with constant velocity $\simeq 100 \mathrm{rad} / \mathrm{s}$ is executed for 23 minutes in order to serve as a data basis, which is independent of the training data of the neural network. The measured and simulated friction torques are presented in Fig. 10 together with the corresponding temperature values. This experiment corresponds to a vertical cross-section in the plot of the training data depicted in Fig. 9. The results emphasize the importance of considering the nonlinear 


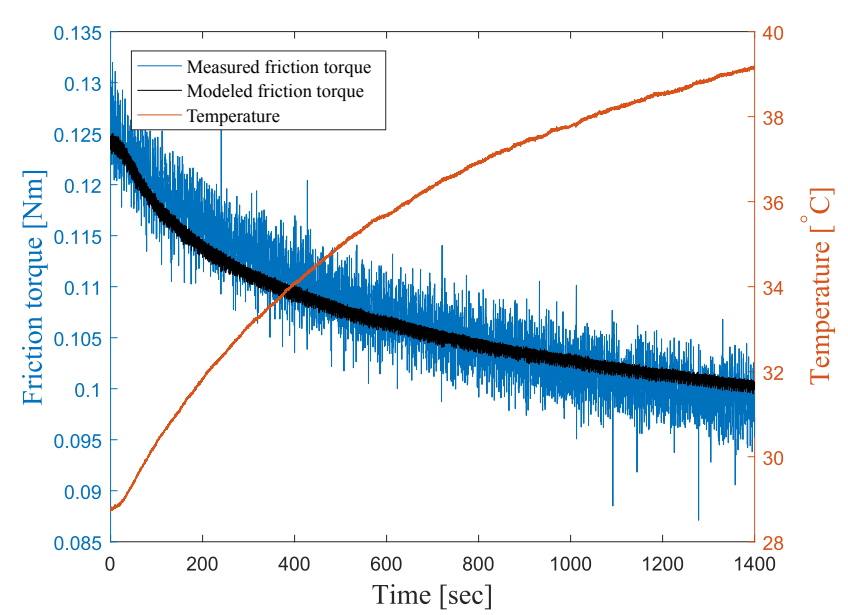

Fig. 10: The measured and simulated friction torques at motor side with continuously increasing temperature at constant velocity of $\simeq 100 \mathrm{rad} / \mathrm{s}$.

temperature dependent friction behavior even at constant velocity. The change in friction torque at $\simeq 100 \mathrm{rad} / \mathrm{s}$ within $10{ }^{\circ} \mathrm{C}$ is about $18 \%$ from the initial value, as can be seen in Fig. 10 .

\section{CONCLUSIONS}

The friction of a brush-less DC motor and a Harmonic Drive gear were experimentally investigated. A nonlinear velocity dependency and a nonlinear temperature dependency are identified as dominant influences on the friction. State of the art friction models do not cover these effects. A refinement of a standard LuGre friction model with the aforementioned nonlinear effects in the gross sliding regime is proposed.

Dynamic models of a RoboDrive ILM85 motor and a HD CSD 25 were built including the refined LuGre friction model. A dedicated testbed was developed and used to identify precisely the parameters of the nonlinear dynamic friction models. Three different cases of velocity dependency are investigated. The new motor model was compared to an unmodified LuGre model and a linear viscous friction model and showed better accuracy as well as better zero velocity crossing behavior. The new model has an MSE of $67 \%$ relative to the standard LuGre model and only $4 \%$ of the linear viscous friction model for a chirp torque input signal. A strong temperature dependency was identified in the HD friction and intensively investigated. The friction torque at maximum velocity decreased to $42 \%$ within a temperature range from 24 to $50^{\circ} \mathrm{C}$. A neural network with 3 neurons was trained to model the space of friction torque over nonlinear inverse temperature dependency and nonlinear viscous friction.

The insights on strong nonlinear temperature dependency lead to the conclusion that temperature sensors on the gearbox can improve the friction modeling and with it the motion control significantly. As a result the robot DLR David will be augmented with temperature sensors on the motors and the gearboxes.
Future work will be on an analytical description of the nonlinearity of the temperature dependency. Investigations on the dependency on external load, e.g. caused by gravitation, will be done. Furthermore, it will include investigations on applications of the new model in friction compensation and output torque estimation.

\section{REFERENCES}

[1] S. Wolf and J.-E. Feenders, "Modeling and benchmarking energy efficiency of Variable Stiffness Actuators on the example of the DLR FSJ," in Intelligent Robots and Systems (IROS), 2016 IEEE/RSJ International Conference on. IEEE, 2016, pp. 529-536.

[2] H. Olsson, K. J. Åström, C. C. De Wit, M. Gäfvert, and P. Lischinsky, "Friction models and friction compensation," European journal of control, vol. 4, no. 3, pp. 176-195, 1998.

[3] R. Mattone and A. De Luca, "Relaxed fault detection and isolation: An application to a nonlinear case study," Automatica, vol. 42, no. 1, pp. 109-116, 2006.

[4] B. Freyermuth, "An approach to model based fault diagnosis of industrial robots," in Robotics and Automation, 1991. Proceedings., 1991 IEEE International Conference on. IEEE, 1991, pp. 13501356.

[5] Harmonic Drive AG, Engineering Data Catalogue, 2017.

[6] T. D. Tuttle and W. P. Seering, "A nonlinear model of a harmonic drive gear transmission," IEEE Transactions on Robotics and Automation, vol. 12, no. 3, pp. 368-374, 1996.

[7] A. Palmgren, Grundlagen der Wälzlagertechnik, 3rd ed. Stuttgart: Frankh'sche Verlagshandlung, 1964, ch. Reibungsmoment der Lager, pp. 39-45.

[8] R. Isermann, Mechatronic systems: fundamentals. Springer Science \& Business Media, 2007.

[9] W. Seyfferth, A. Maghzal, and J. Angeles, "Nonlinear modeling and parameter identification of harmonic drive robotic transmissions," in Robotics and Automation, 1995. Proceedings., 1995 IEEE International Conference on, vol. 3. Nagoya, Japan: IEEE, May 1995, pp. 3027-3032.

[10] T. Tuttle and W. Seering, "A nonlinear model of a harmonic drive gear transmission," IEEE Transactions on Robotics and Automation, vol. 12, no. 3, pp. 368-374, June 1996.

[11] H. D. Taghirad and P. R. Belanger, "An experimental study on modelling and identification of harmonic drive systems," in Proceedings of 35th IEEE Conference on Decision and Control, vol. 4, Dec 1996, pp. $4725-4730$ vol. 4 .

[12] C. C. de Wit, H. Olsson, K. J. Åström, and P. Lischinsky, "A new model for control of systems with friction," Automatic Control, IEEE Transactions on, vol. 40, no. 3, pp. 419-425, Mar 1995.

[13] H. Olsson, "Control systems with friction," Ph.D. dissertation, Lund University, 1996.

[14] A. C. Bittencourt, E. Wernholt, S. Sander-Tavallaey, and T. Brogårdh, "An extended friction model to capture load and temperature effects in robot joints," in Intelligent Robots and Systems (IROS), 2010 IEEE/RSJ International Conference on. IEEE, Oct 2010, pp. 6161-6167.

[15] V. Held and C. Maron, "Estimation of friction characteristics, inertial and coupling coefficients in robotic joints based on current and speed measurements," IFAC Proceedings Volumes, vol. 21, no. 16, pp. 207212, 1988.

[16] B. Armstrong-Helouvry, Control of machines with friction. Springer Science \& Business Media, 2012, vol. 128.

[17] A. Tustin, "The effects of backlash and of speed-dependent friction on the stability of closed-cycle control systems," Electrical Engineers - Part IIA: Automatic Regulators and Servo Mechanisms, Journal of the Institution of, vol. 94, no. 1, pp. 143-151, May 1947.

[18] K. Johanastrom and C. Canudas-De-Wit, "Revisiting the lugre friction model," IEEE control Systems, vol. 28, no. 6, pp. 101-114, 2008.

[19] V. Lampaert, J. Swevers, and F. Al-Bender, "Modification of the Leuven integrated friction model structure," IEEE Transactions on Automatic Control, vol. 47, no. 4, pp. 683-687, Apr 2002.

[20] F. Loeffl, A. Werner, D. Lakatos, J. Reinecke, S. Wolf, R. Burger, T. Gumpert, F. Schmidt, C. Ott, M. Grebenstein, and A. AlbuSchffer, "The DLR C-runner: Concept, design and experiments," in 2016 IEEE-RAS 16th International Conference on Humanoid Robots (Humanoids), Nov 2016, pp. 758-765. 